\title{
Non-Thermal Processes in Cosmological Simulations
}

\author{
K. Dolag • A.M. Bykov • A. Diaferio
}

Received: 17 September 2007 / Accepted: 7 November 2007 / Published online: 14 February 2008

(C) Springer Science+Business Media B.V. 2008

\begin{abstract}
Non-thermal components are key ingredients for understanding clusters of galaxies. In the hierarchical model of structure formation, shocks and large-scale turbulence are unavoidable in the cluster formation processes. Understanding the amplification and evolution of the magnetic field in galaxy clusters is necessary for modelling both the heat transport and the dissipative processes in the hot intra-cluster plasma. The acceleration, transport and interactions of non-thermal energetic particles are essential for modelling the observed emissions. Therefore, the inclusion of the non-thermal components will be mandatory for simulating accurately the global dynamical processes in clusters. In this review, we summarise the results obtained with the simulations of the formation of galaxy clusters which address the issues of shocks, magnetic field, cosmic ray particles and turbulence.
\end{abstract}

Keywords Cosmology: theory, large-scale structure of universe $\cdot$ Acceleration of particles · Hydrodynamics · Magnetic fields · Method: numerical, N-body simulations

\section{Introduction}

Within the intra-cluster medium (ICM), there are several processes which involve nonthermal components, such as the magnetic field and cosmic rays (CRs). At first glance,

\footnotetext{
K. Dolag $(\bowtie)$

Max-Planck-Institut für Astrophysik, P.O. Box 1317, 85741 Garching, Germany

e-mail:kdolag@mpa-garching.mpg.de
}

\section{A.M. Bykov}

A.F. Ioffe Institute of Physics and Technology, St. Petersburg 194021, Russia

e-mail: byk@astro.ioffe.ru

\section{A. Diaferio}

Dipartimento di Fisica Generale "Amedeo Avogadro", Università degli Studi di Torino, Torino, Italy

\section{A. Diaferio}

Istituto Nazionale di Fisica Nucleare (INFN), Sezione di Torino, Via P. Giuria 1, 10125 Torino, Italy 
many of these processes can be studied numerically in simplified configurations where one can learn how the individual processes work in detail. For example, there are investigations which consider magneto-hydro-dynamical (MHD) simulations of cloud-wind interactions (see Gregori et al. 2000, and references therein) or simulate the rise of relic radio bubbles (see Jones and De Young 2005; Reynolds et al. 2005, and references herein). Such work usually focuses on the relevance of CRs, turbulence and the local magnetic fields within and around these bubbles. In this review, we will instead concentrate on simulations of non-thermal phenomena, which aim at understanding the relevance of these phenomena for galaxy cluster properties or at unveiling possible origins of the non-thermal radiation. So far, firm evidence for the presence of non-thermal emission (at radio wavelengths) and for the presence of cosmologically relevant extended magnetic fields has been found only in galaxy clusters; in filaments only weak limits have been found so far. For a more detailed discussion see the reviews on radio observations by Rephaeli et al. (2008) - Chap. 5, this issue, and Ferrari et al. 2008-Chap. 6, this issue.

\section{Possible Origins of Non-thermal Components within the Large-Scale Structure (LSS)}

The origin of magnetic fields and of CRs in galaxy clusters is still under debate. The variety of possible contributors to magnetic fields ranges from primordial fields, battery and dynamo fields to all the classes of astrophysical objects which can contribute with their ejecta. The latter possibility is supported by the observation of metal enrichment of the ICM, which is due to the ejecta of stars in galaxies, see Werner et al. 2008-Chap. 16, this issue, and Schindler and Diaferio 2008-Chap. 17, this issue. The magnetic fields produced by all these contributors will be compressed and amplified by the process of structure formation. The exact amount of amplification and the resulting filling factor of the magnetic field will depend on where and when the contributor is thought to be more efficient.

CRs are produced at shocks, both in supernova remnants and in cosmological accretion and merger shocks. Furthermore, active galactic nuclei (AGN) and radio galaxies contribute to the $\mathrm{CR}$ population. $\mathrm{CR}$ protons can also produce $\mathrm{CR}$ electrons via hadronic reactions with the thermal plasma, leading to the so-called secondary CRs.

\subsection{Possible Origins of Magnetic Fields}

Magnetic fields can be produced either at relatively low redshift $(z \sim 2-3)$ or at high redshift $(z \gtrsim 4)$. In the former case, galactic winds (e.g. Völk and Atoyan 2000) or AGN ejecta (e.g. Enßlin et al. 1997; Furlanetto and Loeb 2001, and references therein) produce magnetic fields 'locally', e.g. within the proto-cluster region. In the latter case, the magnetic field seeds can also be produced by an early population of dwarf starburst galaxies or AGN before galaxy clusters form gravitationally bound systems.

One of the main arguments in favour of the 'low-redshift' models is that the high metallicity observed in the ICM suggests that a significant enrichment occurred in the past due to galactic winds or AGN. Winds and jets should carry magnetic fields together with the processed matter. It has been shown that winds from ordinary galaxies give rise to magnetic fields which are far weaker than those observed in galaxy clusters, whereas magnetic fields produced by the ejecta of starburst galaxies can be as large as $0.1 \mu \mathrm{G}$. Clearly, this class of models predicts that magnetic fields are mainly concentrated in and around galaxies and within galaxy clusters. Note that if the magnetic pollution happens early enough 
(around $z \sim 3$ ), these fields will be amplified not only by the adiabatic compression of the proto-cluster region, but also by shear flows, turbulent motions, and merging events during the formation of the cluster. Shocks are expected to be produced copiously during the nonlinear stage of the LSS formation (see below). Recent detailed studies of shock propagation revealed the presence of specific instabilities driven by energetic accelerated particles (Bell and Lucek 2001; Vladimirov et al. 2006). Such instabilities result in a strong, non-adiabatic amplification of an upstream magnetic field seed which converts an appreciable fraction of the shock ram pressure into magnetic fields.

In the 'high-redshift' models of the magnetic field generation, the strength of the field seed is expected to be considerably smaller than in the previous scenario, but the adiabatic compression of the gas and the shear flows driven by the accretion of structures can give rise to a considerable amplification of the magnetic fields. Several mechanisms have been proposed to explain the origin of magnetic field seeds at high redshift. Some of them are similar to those discussed above, differing only in the time when the magnetic pollution is assumed to take place. In the present class of models the magnetic field seeds are supposed to be expelled by an early population of AGN or dwarf starburst galaxies at a redshift between 4 and 6 (Kronberg et al. 1999); this process would magnetise a large fraction of the volume. Recently, the validity of such a scenario has been confirmed by a semi-analytic modelling of galactic winds (Bertone et al. 2006).

Alternative models invoke processes that took place in the early universe. Indeed, the presence of magnetic fields in almost all the regions of the universe suggests that they may have a cosmological origin. In general, most of the 'high- $z$ models' predict magnetic field seeds filling the entire volume of the universe. However, the assumed coherence length of the field crucially depends on the details of the model. While scenarios based on phase transitions give rise to coherence lengths which are so small that the corresponding fields have probably been dissipated, magnetic fields generated at neutrino or photon decoupling are thought to have a much higher chance of surviving until the present time. Another (speculative) possibility is that the field seed was produced during inflation. In this case, the coherence length can be as large as the Hubble radius. See Grasso and Rubinstein (2001) for a review.

Magnetic field seeds can also be produced by the so-called Biermann battery effect (Kulsrud et al. 1997; Ryu et al. 1998). The idea here is that merger/accretion shocks related to the hierarchical structure formation process give rise to small thermionic electric currents which, in turn, may generate magnetic fields. The battery process has the attractive feature of being independent of unknown physics at high redshift. Its drawback is that, due to the large conductivity of the ICM, it can give rise to very tiny magnetic fields, of order $10^{-21} \mathrm{G}$ at most. One therefore needs to invoke a subsequent turbulent dynamo to boost the field strength to the observed level.

Such a turbulent amplification, however, cannot be simulated numerically yet, making it quite difficult to predict how it would proceed in a realistic environment. It is clear that one expects the level of turbulence to be strongly dependent on the environment, and that it should appear mostly in high-density regions like collapsed objects. While energetic events, such as mergers of galaxy clusters, can easily be effective at driving the required levels of turbulence, it is harder to understand how turbulent amplification would work in relatively quiet regions like filaments. Here, magnetic fields can be rather amplified by the shocks which originate during the LSS formation, as mentioned at the beginning of this section. Lacking a theoretical understanding of the turbulent amplification, it is therefore not straightforward to relate the very weak field seeds produced by the battery process to the 
magnetic fields observed today. Attempts to construct such models, based on combining numerical and analytical computations, have not yet been reported to successfully reproduce the observed scaling relations of the magnetic fields in galaxy clusters.

\subsection{Possible Origins of CRs}

There are many possible contributors to the CR population in the ICM. AGN and stellar activity in cluster galaxies are believed to be a significant source of CRs in the ICM. Furthermore, cluster accretion shocks of high Mach numbers (typically above 100) and of sizes of megaparsec or larger are widely accepted to be possible CR sources with nuclei accelerated up to $\sim 10^{9} \mathrm{GeV}$ (e.g. Norman et al. 1995). Cluster mergers generate internal shocks (of moderate Mach numbers, typically below 4) which provide most of the ICM gas heating (see Kang et al. 2005), but are also likely to convert a non-negligible fraction ( their power into CRs.

AGN outflows dissipate their kinetic energy and Poynting fluxes into the ICM providing additional non-gravitational ICM heating and, thus, a plausible feedback solution to the cooling flow problem (e.g. Binney and Tabor 1995; Churazov et al. 2003). Recent XMMNewton observations of the cluster MS 0735+7421 (Gitti et al. 2007) revealed that a powerful AGN outburst deposited about $6 \times 10^{61}$ erg into the ICM outside the cooling region of radius $\sim 100 \mathrm{kpc}$. Relativistic outflows of AGN are likely to be an essential source of superthermal particles in clusters. A powerful relativistic AGN jet could deposit up to $10^{62} \mathrm{erg}$ into a relativistic particle pool during a duty cycle of about $\sim 50 \mathrm{Myr}$. A potential source of energetic particles are sub-relativistic ions with energy $\sim 100 \mathrm{MeV}$ that are evaporated from an AGN, which accretes mass in the ion-supported tori regime (e.g. Rees et al. 1982). A certain fraction of the energetic particles could escape the flows and avoid fast cooling due to collective effects in the central parts of the cluster. Re-acceleration of that non-thermal population by inner shocks inside the cluster can provide a long-lived non-thermal component that contributes to the total pressure of the ICM.

Star formation activity in galaxies is another source of CRs in clusters of galaxies (e.g. Völk et al. 1996, and references therein). The combined action of supernovae and winds of early-type stars leads to the formation of a hot, X-ray emitting, slowly expanding superbubble filled with large-scale (tens of parsecs) compressible MHD motions (e.g. rarefactions) and shocks. When the starburst event is energetic enough, the superbubble may expand beyond the disk of the parent galaxy and produce a superwind that supplies the intergalactic medium with metals ejected by supernovae and CRs. The superwinds have been seen with Chandra in some starburst galaxies: M 82, Arp 220 and NGC 253. In the superwind scenario, the CR ejection is tightly connected with the metal enrichment of the cluster (Schindler and Diaferio 2008-Chap. 17, this issue).

Bykov (2001) argues that non-thermal particle acceleration can favourably occur in correlated supernovae events and powerful stellar winds with great energy release, which generate interacting shock waves within superbubbles. The acceleration mechanism provides efficient creation of a non-thermal nuclei population with a hard low-energy spectrum; this population can transport a substantial fraction $(\sim 30 \%)$ of the kinetic energy released by the supernovae and by the winds of young massive stars.

A bright phase in the galaxy evolution can be the source of the relic CRs in clusters. Energetic nuclei can be stored in cluster magnetic fields for several Hubble times (e.g. Berezinsky et al. 1997). The presence of these nuclei produces a diffuse flux of high-energy $\gamma$ and neutrino radiation due to the interaction of the CRs with the ICM (Völk et al. 1996; Enßlin et al. 1997). The resulting flux depends on the ICM baryon density. 
A physical model of particle acceleration by the ensemble of inner shocks in the ICM could be similar to the superbubble model. The sub-cluster merging processes and the supersonic motions of dark matter halos in the ICM are accompanied by the formation of shocks, large-scale flows and broad spectra of MHD-fluctuations in a tenuous intra-cluster plasma with frozen-in magnetic fields. Vortex electric fields generated by the large-scale motions of highly conductive plasma harbouring shocks result in a non-thermal distribution of the charged nuclei. The free energy available for the acceleration of energetic particles is in the ram pressure of the shocks and in the large-scale motions.

The most studied way to transfer the power of the MHD motions to the energetic particle population is the Fermi-type acceleration (see e.g. the review by Blandford and Eichler 1987 and Bykov et al. 2008 - Chap. 7, this issue). An important ingredient of the energetic particle acceleration by shocks and large-scale MHD motions is the presence of small-scale MHD turbulence, which is necessary to scatter relativistic particles and to make their pitch-angle isotropic. The scale of the fluctuations required for the resonant scattering of a particle of energy $\sim 1 \mathrm{GeV}$ is about $3 \times 10^{12} B_{-6}^{-1} \mathrm{~cm}$, where $B_{-6}$ is the local mean magnetic field in $\mu \mathrm{G}$. The scale is some 10-11 orders of magnitude smaller than the basic energy scale of the system; thus the origin and the maintenance mechanisms of such small scale turbulence is a serious problem.

In non-linear models of particle acceleration by strong MHD shocks the presence of turbulence could be supported by the CR instabilities themselves (see e.g. recent non-linear Monte-Carlo simulations by Vladimirov et al. 2006). Direct MHD cascade of energy to small scale turbulence is also possible, but the small scale fluctuations are highly anisotropic in that case (see e.g. Biskamp 2003). Moreover, the cascade properties are still poorly known at the scales close to the Coulomb mean free path where the transition from the collisional to the collisionless plasma regime occurs.

The particle distribution within a system with multiple shocks and with large-scale plasma motions is highly intermittent, because it has strong peaks at shocks and a nonsteady mean CR background. By using the kinetic equations given by Bykov (2001), one can construct a model of the temporal evolution of the particle distribution function, which accounts for the non-linear effect of the reaction of the accelerated particles on the shock turbulence inside the cluster. In Fig. 1 we show the efficiency of a conversion into CRs of the power of shocks and the bulk plasma motions of a scale of about $100 \mathrm{kpc}$. One may note that, while the efficiency could be as high as $30 \%$ for some relatively short period of time, the efficiency is typically $\sim 10 \%$ for most of the time. Fig. 2 shows the temporal behaviour of the particle spectra on a Gyr time scale.

Non-thermal particles and magnetic fields could contribute to the total pressure of the ICM. In the particular model described above a substantial energy density could be stored in protons of energy below $200 \mathrm{MeV}$. Protons of that energy level produce very little high energy emission and therefore it is rather difficult to constrain that component directly from the observational data which are currently available.

\section{Shocks in the LSS and Clusters}

As described in the previous section, shocks can play a crucial role as source of non-thermal components in galaxy clusters. Therefore, a significant effort is spent to investigate the distribution and the evolution of shocks during structure formation.

In early simulations of the formation of cosmological structures, it was already noticed that shocks are the main source of the ICM heating (see Quilis et al. 1998, and references therein). In numerical simulations, two classes of shocks can be identified. 


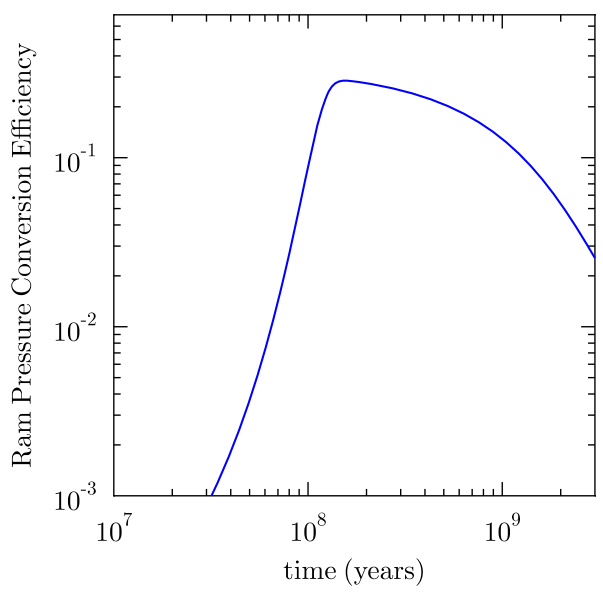

Fig. 1 The simulated efficiency of conversion of shock and bulk MHD motions ram pressure power to the CRs at different stages of cluster evolution. The efficiency is defined as a volume averaged fraction of the cluster large-scale motions power transferred to CRs. The simulation was done for a model of CR acceleration by an ensemble of shocks and MHD-turbulent motions described in Bykov (2001), adopted for clusters of galaxies. The MHD turbulence in a cluster of a Mpc size was initiated with the Gaussian bulk velocity dispersion of $2000 \mathrm{~km} \mathrm{~s}^{-1}$ at a scale of $100 \mathrm{kpc}$

Fig. 2 The temporal evolution of the particle distribution function in a cluster of a Mpc size and a Gaussian bulk velocity dispersion of about $2000 \mathrm{~km} \mathrm{~s}^{-1}$ at a scale of $100 \mathrm{kpc}$. The curves labelled as 1,2, 3 correspond to $0.1,0.3$ and $1 \mathrm{Gyr}$ age of the cluster. Mono-energetic proton injection at energy $10 \mathrm{keV}\left(p_{0}\right.$ is the injection momentum). The injection efficiency (see Fig. 1) was assumed to be about $10^{-3}$. The power conversion efficiency for the model was already illustrated in Fig. 1

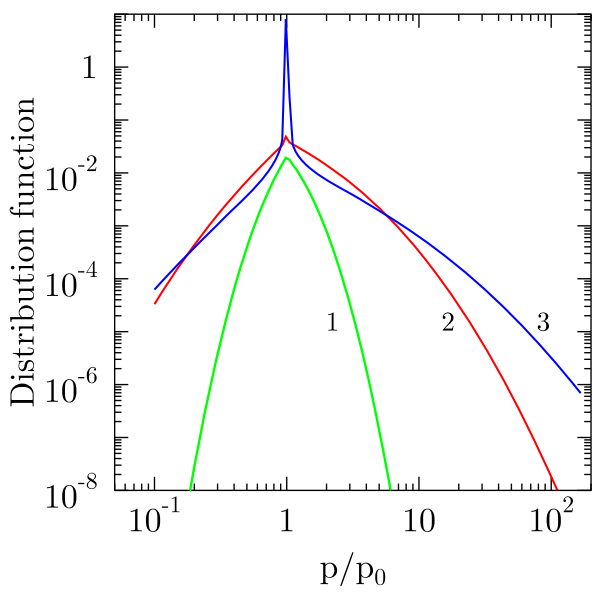

The first one is the accretion shocks surrounding the forming objects, namely filaments or galaxy clusters. In the case of galaxy clusters, most shocks are quasi-spherical and their position with respect to the centre of the cluster grows with time (shock travelling outwards). These shocks can be associated with the accretion of diffuse matter which is relatively cold. These shocks provide a first step towards the virialisation of an initially supersonic accreting flow. The radius of the accretion shock (for a quasi-spherical cluster at $z=0$ ) is typically two times the virial radius. As the typical temperature and density of the upstream gas is quite low, such shocks can have very large Mach numbers ( $\gg 10)$.

The second class of shocks in galaxy clusters arises from the merging of substructures. The gas in gravitationally bound substructures is much denser than the diffuse ICM; there- 
Fig. 3 Shocks detected in a cosmological simulation of a cubic volume with a side length of $100 \mathrm{Mpc}$, resolved with $512^{3}$ grid points. The figure shows the kinetic energy flux per unit comoving volume through surfaces of external and internal shocks with Mach number between $\log M$ and $\log M+\mathrm{d}(\log M)$ at different redshifts (solid, $z=0$ to dotted, $z=2)$. From Ryu et al. (2003)

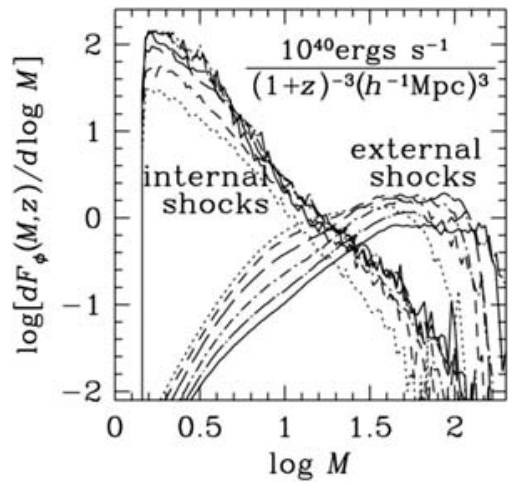

fore it cannot be stopped in the accretion shock and it continues to move with the substructure through the gas, which is mostly virialised. The substructures, which have velocities larger than the sound velocity of the hot ICM, drive the so-called merger shocks. These shocks are initiated within the ICM which has been already shock-heated, and therefore they typically have modest Mach numbers (below $\approx 4$ ). The merger shocks propagate within the dense ICM and are likely to be the main contributor to the global energy dissipation within the ICM (see Pfrommer et al. 2006, and references therein). As these shocks are primarily driven by gravitational attraction, non-gravitational processes (like cooling or feedback by star formation processes) do not significantly affect the global energy dissipation (see Kang et al. 2007). The ram pressure dissipation process in shocks within the collisionless plasma is non-trivial because of the role of long-lived non-thermal components, namely magnetic fields, MHD-waves and energetic particles (see for details Bykov et al. 2008 - Chap. 7, this issue). The complex multi-scale structure of a shock modified by energetic CRs (e.g. where the back reaction of the CRs on the thermal plasma is strong enough to change the thermodynamical states of the plasma in addition to the shock itself) cannot be resolved properly in LSS simulations.

The efficiency of energising CRs by shocks strongly depends on a number of factors. Among the most important factors, we have (i) the upstream magnetic field of the shock, (ii) the plasma parameter $\beta=\mathcal{M}_{\mathrm{a}}^{2} / \mathcal{M}_{\mathrm{s}}^{2}$ (ratio of thermal to magnetic pressure, here expressed by the Mach numbers $\mathcal{M}_{\mathrm{a}}$ and $\mathcal{M}_{\mathrm{s}}$ with respect to the Alfvén and sound velocity respectively), (iii) the inclination of the upstream field of the shock, and (iv) the presence of a pre-existing CR population produced by the accretion shock or in star-forming regions.

The distribution of the Mach numbers of the shocks within the cosmological structure can be derived from semi-analytical modelling (see Gabici and Blasi 2003; Pavlidou and Fields 2006, and references therein), as well as directly from cosmological simulations (see Miniati et al. 2000; Ryu et al. 2003; Pfrommer et al. 2006; Kang et al. 2007, and references therein). An example is shown in Fig. 3. There are significant differences in the strength and the distribution of the shocks found in different works: this fact has strong consequences on the predicted amount and the relative importance of CRs and their energy with respect to the thermal energy of the ICM. Note that part of the confusion, specially in the early work, is caused by a lack of resolution within the simulations, which prevents internal shocks from being properly resolved (see Ryu et al. 2003; Pfrommer et al. 2006); further confusion originates from the influence of other, non-thermal processes (like preheating) on the inferred Mach number of the external shocks (see Pfrommer et al. 2006; Kang et al. 2007). Moreover, differences in the underlying hydrodynamic simulations as well as in the shock detection, mostly done in a post processing fashion, can contribute to 


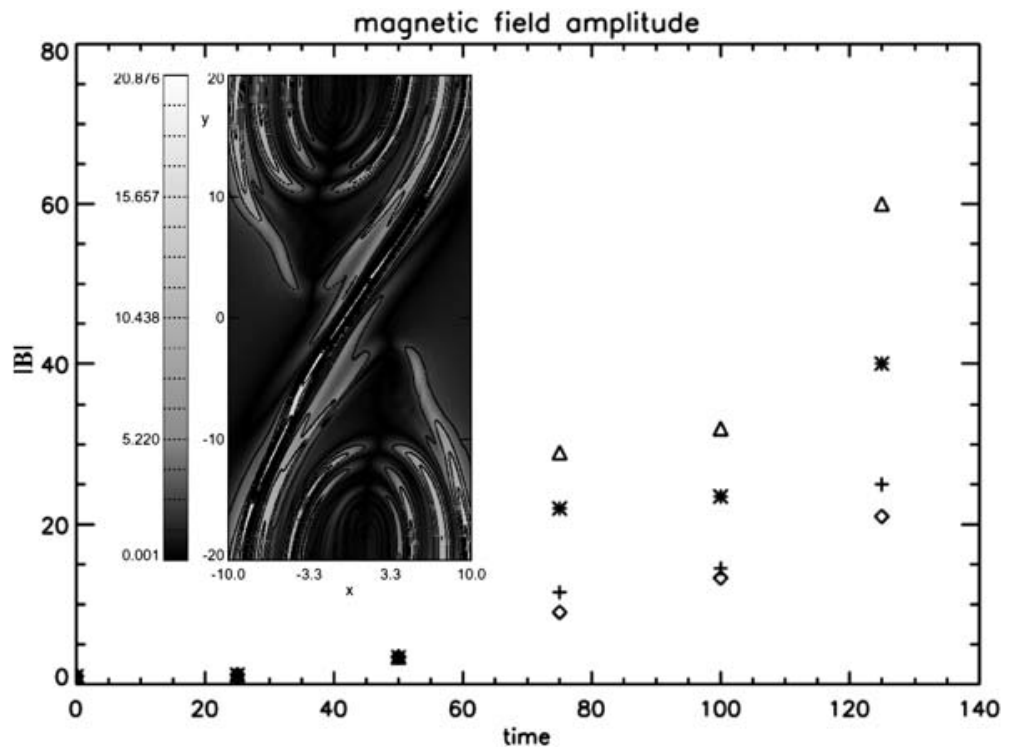

Fig. 4 Resistive, MHD simulation of a flow which is KH instable at the interface separating the left and right halve of the simulated volume. Shown is the magnetic field (y-component) in units of the initial magnetic field after evolving the $\mathrm{KH}$ instability (inlay) and the time evolution of the (relative) magnetic field amplitude for different values of the chosen resistivity and initial magnetic field. Time is in units of the Alfvénic transit time (4000 year). Triangles and stars are for an initial magnetic field of $10 \mu \mathrm{G}$, crosses and diamonds for a ten times larger field. Stars and diamonds have a magnetic Reynolds number of 100, triangles and crosses have a ten times larger magnetic Reynolds number. From Birk et al. (1999)

differences in the results. Nevertheless, there is a qualitative agreement in the case of simulated internal shocks between the simulations done by Ryu et al. (2003) and Pfrommer et al. (2006).

\section{Evolution of Magnetic Fields in Simulations}

\subsection{Local Amplification of Magnetic Fields}

A very basic process which amplifies magnetic fields is related to the Kelvin-Helmholtz $(\mathrm{KH})$ instabilities driven by shear flows, which are common during the formation of cosmic structures. Birk et al. (1999) performed a detailed study of such an amplification within the outflows of starburst galaxies, where the KH timescale should be $\approx 4 \times 10^{5}$ years (see Fig. 4). By using a Cartesian resistive MHD code they found that the amplification factor of the magnetic field mainly depends on the initial ratio of the magnetic to the kinetic energy and only mildly depends on the assumed resistivity. They concluded that such a process could indeed explain why the magnetic field observed in the halo of starburst galaxies is significantly higher than what is expected from the magnetic fields observed within galactic disks. When applied to a cluster core environment, the $\mathrm{KH}$ timescale turns out to be $10^{7}$ years; this timescale makes the $\mathrm{KH}$ instability an interesting process to amplify weak magnetic fields.

It is quite expected that merger events and accretion of material onto galaxy clusters will drive significant shear flows within the ICM. Extensive MHD simulations of single 


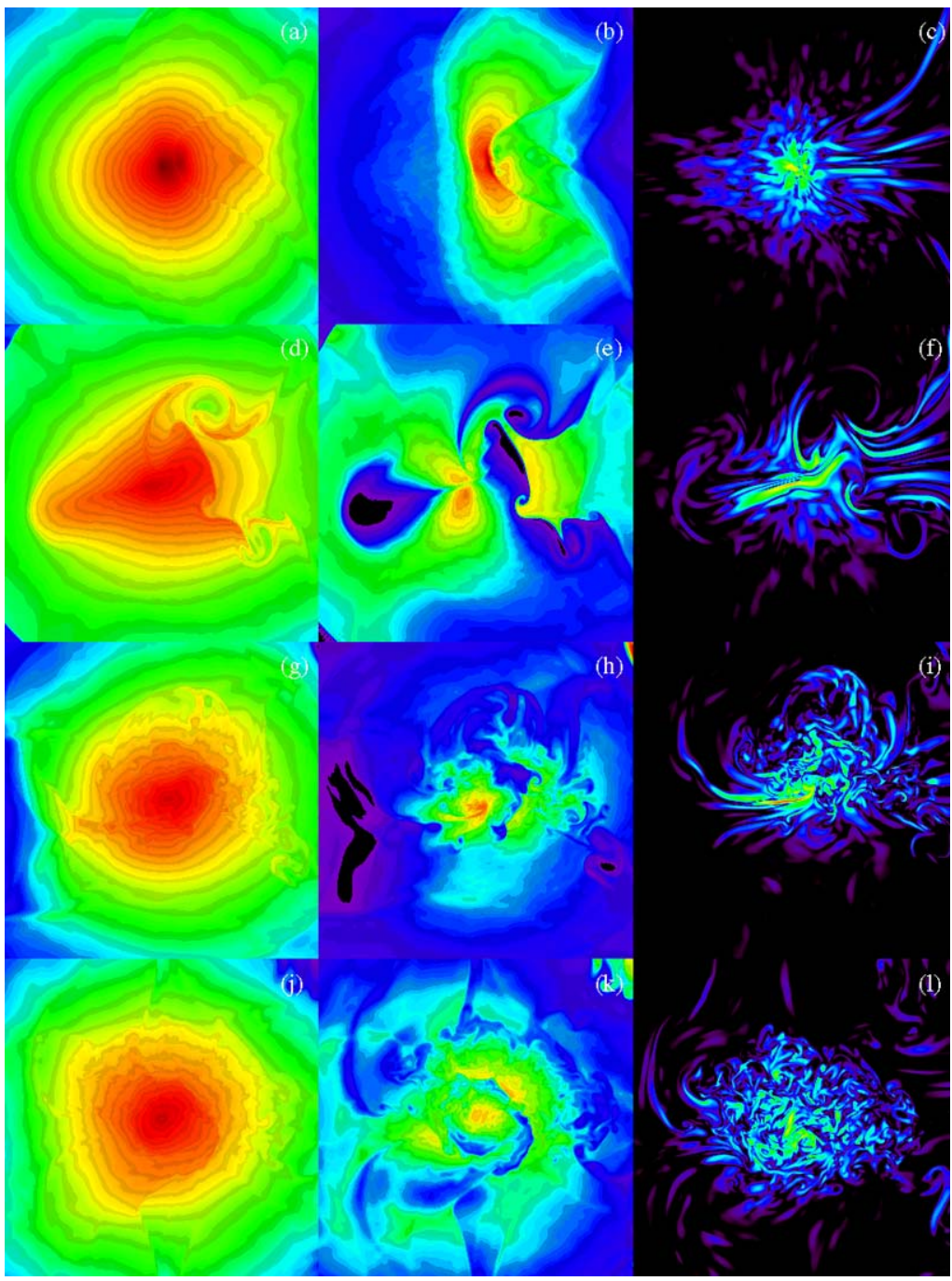

Fig. 5 The evolution of (the logarithm of) the gas density (left column), the gas temperature (central column), and (the logarithm of) the magnetic pressure (right column) in two-dimensional slices taken through the core of a cluster in a major merger phase in the plane of the merger. Each row refers to different epochs: $t=0$ (i.e. the time of the core coincidence), $t=1.3, t=3.4$, and $t=5.0 \mathrm{Gyr}$, from top to bottom. Each panel is $3.75 \times 3.75 \mathrm{Mpc}$. The merging subcluster enters from the right. From Roettiger et al. (1999b)

merging events performed using the Eulerian code ZEUS (Stone and Norman 1992a, 1992b) demonstrated that merger events are effective at amplifying magnetic fields (Roettiger et al. 1999b). In particular these authors found that the field initially becomes quite filamentary, as a result of stretching and compression caused by shocks and bulk flows during infall; at this stage only a minimal amplification occurs. When the bulk flow is replaced by turbulent motions (e.g., eddies), the field amplification is more rapid, particularly in localised regions, see Fig. 5. The total magnetic field energy is found to increase by nearly a factor of three 


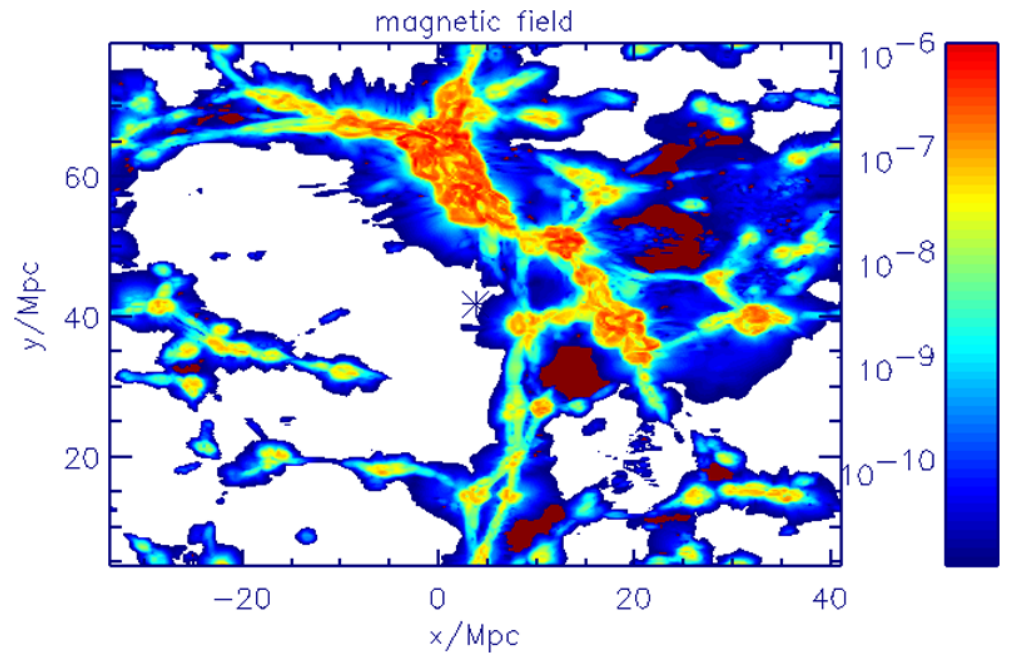

Fig. 6 A two-dimensional cut through a cosmological box simulated by Miniati et al. (2001), following the evolution of battery fields. The logarithm of the up-scaled magnetic field strength in Gauss at $z=0$ is shown. Taken from Sigl et al. (2004)

with respect to a non-merging cluster. In localised regions (associated with high vorticity), the magnetic energy can increase by a factor of 20 or more.

A power spectrum analysis of the magnetic energy showed that the amplification is largely confined to scales comparable to or smaller than the cluster cores: this indicates that the core dimensions define the injection scale. It is worth taking notice that the previous results can be considered as a lower limit on the total amplification, because of the lack of resolution. Furthermore, it is quite likely that a galaxy cluster undergoes more than one of these events during its formation process, and that the accretion of smaller haloes also injects turbulent motions into the ICM: consequently, the magnetic field amplification within galaxy clusters will be even larger. A detailed discussion of the amplification of magnetic field in the cluster environment, using various simulations of driven turbulence, can be found in Subramanian et al. (2006); these authors show that turbulent processes can provide reasonable strength and length scales of the magnetic fields in galaxy clusters.

\subsection{Magnetic Fields From Cosmological Shocks}

Cosmological shocks, mainly the accretion shocks on cosmological objects like galaxy clusters and filaments, occur more frequently than the ones produced by individual merger events. They also can produce magnetic fields through the so-called Biermann battery effect (Kulsrud et al. 1997; Ryu et al. 1998), on which a subsequent turbulent dynamo may operate (see Sect. 2).

In such a scenario, the magnetic field is strongly correlated with the large-scale structure (see Fig. 6). This means that the magnetic field within the filamentary structure could be even slightly higher than its equipartition value without violating the (weak) upper limits of the rotation measure (RM) of quasars, as pointed out by Ryu et al. (1998). It is worth pointing out that the arguments for the turbulent dynamo action, which could amplify the battery field seeds up to the $\mu \mathrm{G}$ level, as presented in Kulsrud et al. (1997), refer explicitly to regions which are close to collapse into galaxies. It has still to be proven that such arguments hold 


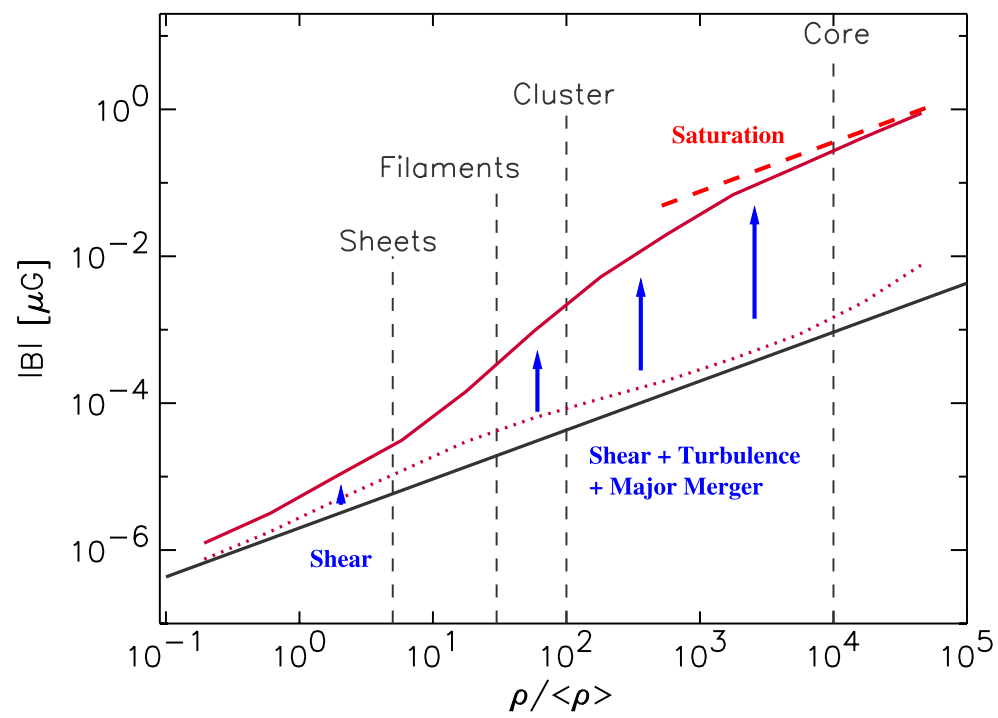

Fig. 7 The strength of the magnetic field as a function of baryonic overdensity within a cosmological simulation. The straight line shows the expectation for a purely adiabatic evolution, the solid line gives the mean field strength at a given overdensity within a cosmological simulation (Dolag et al. 2005a). While the latter is close to the adiabatic value in underdense regions, in clusters there is a significant inductive amplification due to shear flows and turbulence; this amplification however saturates in the cluster cores. At any given density, a large fraction of particles remains close to the adiabatic expectation, as shown by the dotted line, which gives the median of the distribution at each density

within proto-clusters or even cosmological structures, like sheets and filaments. In general, the time evolution of the magnetic field, as predicted by these simulations, strongly flattens around $z \approx 3$ (Kulsrud et al. 1997); the evolution then leads to a magnetic field intensity which is relatively uniform on scales of tens of Mpc within the LSS around galaxy clusters (see Fig. 6). Note that, so far, the synthetic rotation measures due to the magnetic fields predicted by up-scaling the battery fields have never been compared with the rotation measure observed on the scale of galaxy clusters. This might be partially motivated by the lack of resolution in the mock clusters within such non-adaptive, Eulerian simulations.

\subsection{Cosmological MHD Simulations}

By using the GrapeMSPH code (Dolag et al. 1999) and assuming that a small initial magnetic field seed exists before structure formation (see Sect. 2), the first self-consistent simulations which follow the magnetic field amplification during the formation of galaxy clusters within a cosmological environment have been performed by Dolag et al. (1999, 2002). These simulations demonstrated that the contribution to the amplification of magnetic fields by shear flows (and by its induced turbulence) is significant (see Fig. 7). Therefore, for the first time, a consistent picture of the magnetic field in galaxy clusters could be constructed: the amplification predicted by the simulations was capable of linking the predicted strength of the magnetic field seed (see Sect. 2 and references therein) at high redshift $(z \approx 3$ and higher) to the observed magnetic field strength in galaxy clusters in the local universe.

Furthermore, the simulations predicted that the final structure of the magnetic field in galaxy clusters reflects the process of structure formation, and no memory on the initial 


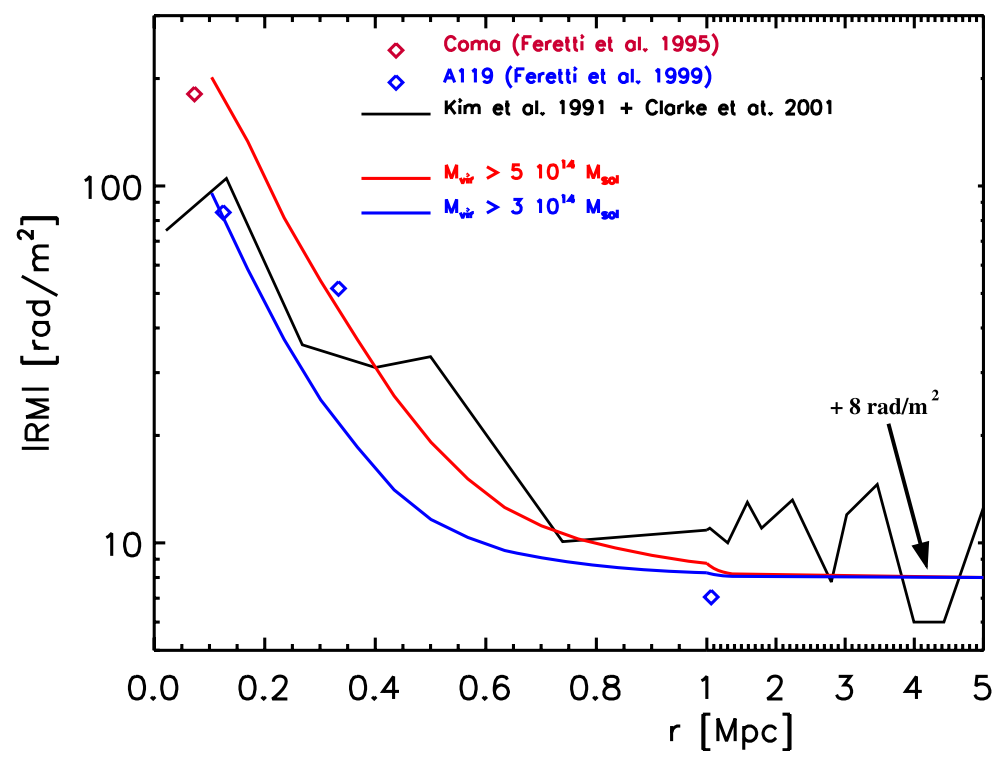

Fig. 8 Comparison of RMs in simulations with observations of Abell clusters, as a function of the distance from the cluster centre. The smooth lines represent the median values of $|\mathrm{RM}|$ produced by simulated clusters with masses above $5 \times 10^{14} \mathrm{M}_{\odot}$ (upper line) and $3 \times 10^{14} \mathrm{M}_{\odot}$ (lower line). The broken line represents the median of combined data taken from the independent samples presented in Kim et al. (1991) and Clarke et al. (2001). We also include data (diamonds) for the three elongated sources observed in A119 (Feretti et al. 1999), and for the elongated source observed in the Coma cluster (Feretti et al. 1995)

magnetic field configuration survives: this relaxes the constraints on models for magnetic field seeds. In general, such models predict a magnetic field profile similar to the density profile. Thereby the predicted rotation measure (RM) profile agrees with the observed one (see Fig. 8). Dolag et al. (2001) found a quasi linear correlation between two observables, namely the X-ray surface brightness and the RM r.m.s., both in observations and in their MHD simulations. By extending the GADGET2 code to follow the full set of ideal MHD equations, Dolag et al. (2004, 2005a) performed several realisations of a cosmological volume which confirm these results at even much higher resolution.

Recently, Brüggen et al. (2005) confirmed all the previous results, which are based on SPH codes, with a simulation of the formation of a single galaxy cluster in a cosmological framework, using a passive MHD solver implemented into FLASH, an adaptive mesh refinement (AMR) code (see Fig. 9).

Another interesting quantity to look at is the slope $\alpha$ of the magnetic field power spectrum ( $\propto k^{-\alpha}$, with $k$ being the wave vector). Within galaxy clusters, $\alpha$ is predicted by the SPH simulations (Dolag et al. 2002; Rordorf et al. 2004) to be slightly lower, but still very close to $11 / 3$, which is the expected value for a Kolmogorov-like spectrum in 3D. The AMR simulation by Brüggen et al. (2005) nearly perfectly matches the Kolmogorov slope.

\subsection{Approximative Simulations}

The fully consistent cosmological MHD simulations described above are very expensive in their requirements of computing resources; thus, for the investigation of various models, simplified approaches are often used in astrophysics. Recently, Bertone et al. (2006) used 


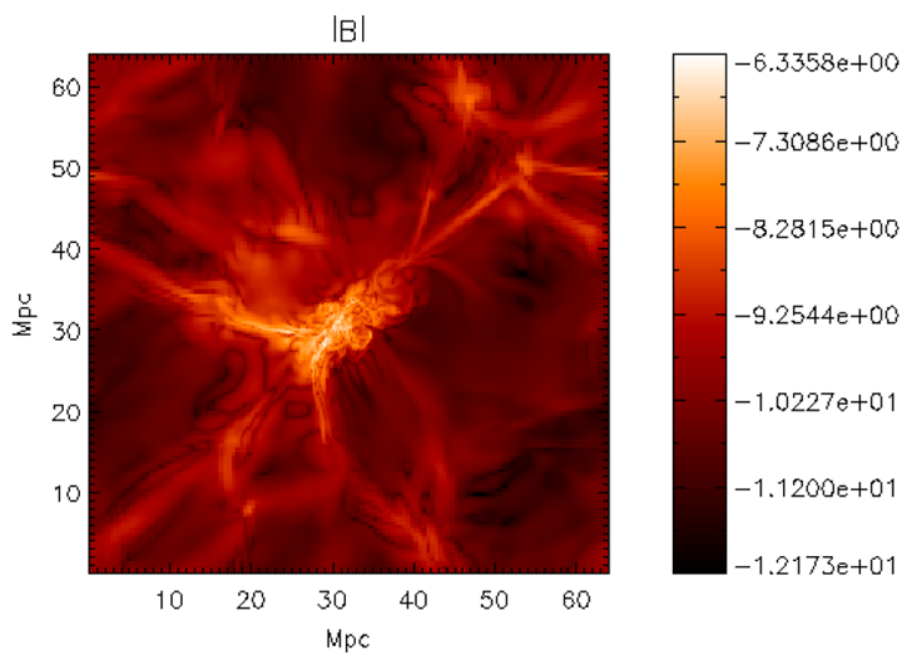

Fig. 9 Slice through the centre of the simulated box hosting a massive galaxy cluster. The simulation used the AMR code FLASH and followed the evolution of a weak magnetic field seed. The figure shows the logarithm of the magnetic field strength, in Gauss, measured at $z=0.5$. From Brüggen et al. (2005)

semi-analytic simulations of magnetised galactic winds coupled to high-resolution N-body simulations of structure formation to estimate limits for the fraction of the ICM which can be significantly magnetised. Interestingly, the fraction of the volume of the ICM, which is predicted to be magnetised at a significant level by the galactic outflows during structure formation, is very high. In fact, the volume filling is high enough to be compatible with observations and a moderately efficient turbulent dynamo, operating within the ICM, will easily amplify these fields up to the observed values.

Additional to any dynamo action, further support for strong magnetic field amplification during the process of structure formation comes from the application of the Zel'dovich approximation (Zel'dovich 1970) to follow the MHD equations during the gravitational collapse (Bruni et al. 2003; King and Coles 2006). These papers demonstrate the existence of a super-adiabatic amplification due to the anisotropy of the collapse of the LSS within the cold dark matter paradigm.

A novel aspect of including magnetic field pressure into LSS simulations-even if in a simplified way - is investigated by Gazzola et al. (2007), which followed a purely empirical approach. Inspired by the scaling between magnetic field and gas density, found in both simulations and observations (Dolag et al. 2001), the magnetic field is parameterised as $B(z)=B_{0}(1+z)^{3 \alpha} \rho^{\alpha}$. Then an isotropic pressure $\left(\propto B^{2}\right)$ term is added into the equation of motion. This approach allows a quite effective way to identify which models of magnetic fields, along with their parameters, provide a non-thermal pressure support which is able to modify significantly the formation of structures. Thereby this approach gives a unique insight for the possible consequences of certain magnetic field scenarios.

\section{Cosmic Rays}

\subsection{Modelling of Cosmic Rays (CRs)}

CRs may originate in a number of cosmological processes including jets due to mass ac- 
cretion, large-scale shocks and supernova remnants (see Sect. 2). One can conventionally distinguish two important classes of CR modelling in cosmological simulations.

The first one is related to the direct modelling of the origin, spatial distribution and spectral evolution of energetic electrons and positrons, which are responsible for most of the non-thermal emission observed so far in cosmological objects. In the vast majority of the non-thermal lepton $\left(\mathrm{e}^{ \pm}\right)$simulations, $\mathrm{e}^{ \pm}$are considered as test particles scattered and accelerated by electro-magnetic fields associated with the cosmological flows. In the highly relativistic energy regime, the leptons radiate very efficiently and therefore suffer from radiative losses. Most of the models are based on the linear kinetic equations for the electron distribution function without accounting for the back-reaction that the accelerated electrons have on the bulk plasma motions and on the MHD-turbulence.

The second class of the CR simulations is related to the acceleration and propagation of energetic baryons. The radiation inferred from the energetic trans-relativistic baryons is basically caused by their nuclear interactions with the ambient matter which produces $\gamma$-rays, neutrino and $\mathrm{e}^{ \pm}$-pairs. Non-relativistic super-thermal baryons can produce some emission by inverse-Bremsstrahlung mechanisms. The most important feature of that class of processes, however, is that energetic non-thermal baryons could absorb a substantial fraction of the ram pressure of both non-relativistic and relativistic plasma flows. The back-reaction effects of energetic non-thermal particles on the plasma flows must then be taken into account. The most popular example of such a non-linear process is the diffusive particle acceleration by astrophysical shocks (see e.g. Blandford and Eichler 1987; Malkov and Drury 2001, for a review). The process of diffusive particle acceleration by MHD shocks could be very efficient in highly turbulent cosmic plasma.

In Fig. 1 of Sect. 2 we illustrated the simulated efficiency of the conversion into CRs of the power of shocks and of the bulk plasma motions in the particular cosmological context which represents the typical environment within galaxy clusters. The non-linear effect of the back-reaction of accelerated particles on large-scale plasma flows results in the specific temporal evolution of the particle spectra shown in Fig. 2 of Sect. 2. Moreover, as it was mentioned above, under a certain condition, thanks to the conversion of a fraction of the shock ram pressure into magnetic field energy, an efficient acceleration of baryons by MHD shocks in a turbulent cosmic plasma results in a strong amplification of the magnetic field in the shock upstream.

The acceleration of relativistic electrons and their emission were modelled in different cosmological contexts. To study the gradients of the spectral indices inferred from the radio observations at 1.4-5.0 GHz of five luminous 3C radio galaxies, Myers and Spangler (1985) developed a model in which the radiation is due to an isotropic ensemble of relativistic electrons which are subject to synchrotron radiation losses (synchrotron aging). Myers and Spangler (1985) inferred the age of the electrons, namely the time since their acceleration, at various locations in the lobes, and consequently derived the speed of the separation of the hot spots from the lobe material. The inferred speeds were found to be in the range 10000 $30000 \mathrm{~km} \mathrm{~s}^{-1}$. These results are consistent with the beam model, in which the lobe material is left behind by a hot spot advancing through the intergalactic medium at speeds of about $10000 \mathrm{~km} \mathrm{~s}^{-1}$.

Enßlin and Gopal-Krishna (2001) modelled different scenarios for the evolution of the radio plasma inside the cocoons of radio galaxies, after the activity of the central engine has ceased. These authors discussed an analytical model for the evolution of a relativistic electron population under synchrotron, inverse Compton and adiabatic energy losses or gains. It was demonstrated that fossil radio plasma with an age of up to 2 Gyr can be revived by compression in a shock wave of large-scale structure formation, caused during the merger 
events or the mass accretion of galaxy clusters. The scenario was applied to explain the origin of the diffuse radio emission found in clusters of galaxies, without any likely parent radio galaxy seen nearby. The model predicts the existence of a population of diffuse radio sources, emitting at very low frequencies with an ultra-steep spectrum, which are located inside and possibly outside galaxy clusters. Thereby, extended radio emission could be interpreted as tracing the revival of aged fossil radio plasma which is caused by the shock waves associated with large-scale structure formation.

Some processes related to CRs were implemented in cosmological simulation codes. For instance, COSMOCR is a numerical code for the investigation of CRs in computational cosmology (Miniati 2001). The code includes a number of prescriptions to account for the diffusive shock acceleration, the mechanical and radiative energy losses and the spatial transport of the energetic particles into the cosmic environment. Primary CR electrons and ions are injected at the shock sites according to the phenomenological thermal leakage prescription. Secondary electrons are continuously injected as a results of proton-proton (p-p) inelastic collisions of primary CR ions and thermal background nuclei. The code consists of a conservative, finite volume method with a power-law sub-grid model in momentum space. Note that in this numerical approach, the back-reaction of the non-thermal components (CRs and magnetic fields) caused by their pressure contribution to the thermal gas is neglected.

To study the impact of CRs on galaxy and cosmic structure formation and evolution, Enßlin et al. (2007) developed an approximative framework which treats dynamical and radiative effects of CRs in cosmological simulations. These authors approximate the CR spectrum of each fluid element by a single power-law, with spatially and temporally varying normalisation, low-energy cut-off, and spectral index. In this framework they included some approximate prescriptions for CR injection and acceleration by shocks, as well as CR transport and energy losses due to Coulomb interactions, ionisation losses, Bremsstrahlung losses, and hadronic interactions with the background matter. These prescriptions are suited to be included into the global schemes of the numerical simulations of galaxy and structure formation. Although in such implementation the description of the CR population is more simplistic than in the work described earlier, the dynamical influence of the CRs onto the underlying hydrodynamics is no longer neglected. This is not only important for the dynamics of the ICM itself but also for the injection of the CRs by shocks, which are altered by the presence of the non-thermal pressure support of the CRs.

\subsection{The Quest for Radio Relics}

Roettiger et al. (1999a) were able to reproduce the main features of the extended peripheral radio emission (the so-called radio relics) observed in A 3667 by combining the single merger simulations (Sect. 4.1) with a model for the in situ re-acceleration of the relativistic particles, as described in the previous section. In their models, they injected, into the ICM, relativistic electrons with a power-law spectrum, where the power-law index $\gamma=3 /(r-1)+1$ is related to the gas compression ratio $r=\rho_{2} / \rho_{1}$ at the shock. They also related the age of the radio plasma $t_{\mathrm{a}}$ to the distance $d=\kappa v_{\mathrm{s}} t_{\mathrm{a}}$, using a weak-field/highdiffusion limit $\kappa=1$. By having effective shock velocities $v_{\mathrm{s}} \approx 700-1000 \mathrm{~km} \mathrm{~s}^{-1}$ and by aging the synchrotron spectrum with the formalism of Myers and Spangler (1985), they were able to reproduce the observed distribution of the spectral index for a magnetic field of $\approx 0.6 \mu \mathrm{G}$ at the position of the radio relic (Fig. 10). Since such configurations seem to be quite common in galaxy clusters, it naturally raises the question, why not all the clusters show such peripheral radio emission. One possible explanation is that such shock structures are relatively short lived compared to the merger event itself. Also, it is necessary to have the 


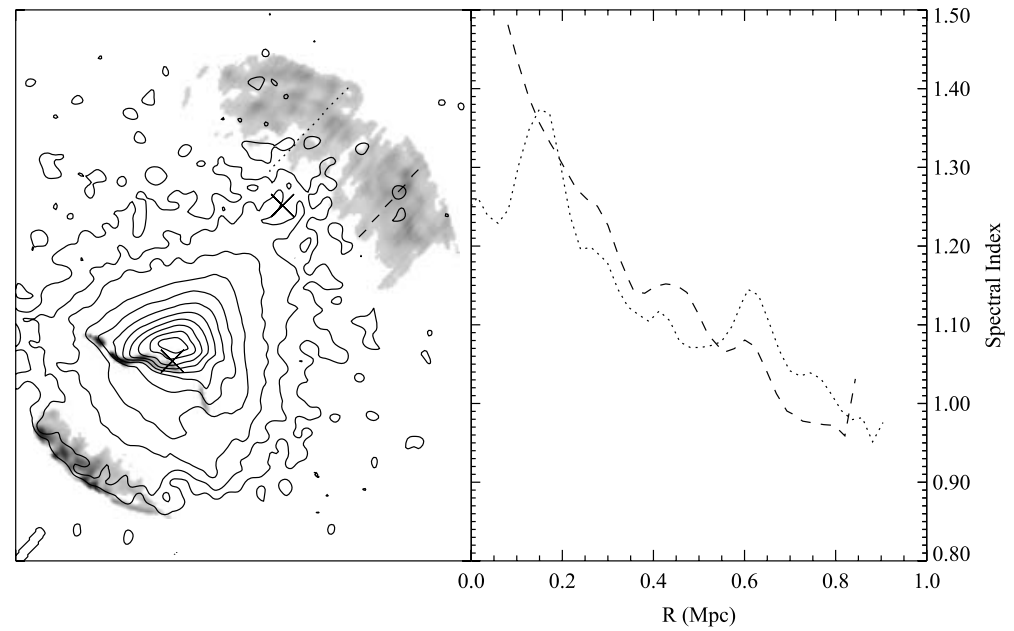

Fig. 10 Results from a simulation intended to reproduce the merging system observed in A 3667 . The left panel shows the simulated X-ray surface brightness (contours) and the radio emission at $1.4 \mathrm{GHz}$ (grey scale). The image is $3.15 \times 3.85 \mathrm{Mpc}$. The dashed and dotted lines refer to the location of the radio spectral index $\left(\alpha_{4.9}^{1.4}\right)$ profiles displayed in the right panel. From Roettiger et al. (1999a)

presence of a large-scale magnetic field. It could further be that only massive clusters can provide enough magnetic field and strong enough merger events to trigger such a peripheral emission.

To overcome this problem, Enßlin and Brüggen (2002) proposed that such radio relics could be made by a pre-existing fossil radio plasma illuminated by the shock waves that the merger events originate. In their models, following Enßlin and Gopal-Krishna (2001) which take into account synchrotron, inverse Compton and adiabatic energy losses and gains, they evolved the electron spectrum for the tracer particles, which represents the fossil radio plasma. Their simulation, using the ZEUS code, follows the evolution of a sphere of tracer particles hit by a shock front (Fig. 11). Such a configuration nicely reproduces the filamentary radio emission and toroidal structures observed in many cases. These simulations also predict that the magnetic fields are mostly aligned with the direction of the filaments, as suggested by observational data.

Hoeft et al. (2004) investigated this idea in a more realistic modelling by using the SPH code GADGET (Springel et al. 2001) to simulate a merging galaxy cluster within a cosmological environment. Such a simulation showed that the probability for a shock wave to flare the radio plasma is highly suppressed in the central regions of galaxy clusters, unlike the peripheral regions, where they found illuminated structures of size up to Mpc scales (Fig. 12). The reason for this is that the radio plasma ages much faster in the cluster centre than in the outer regions. In fact, in the cluster centre, the pressure of the radio plasma is higher and its energy losses due to the higher magnetic field are larger; moreover, the compression ratio of the shock wave is much higher in the low-density peripheral regions than in the cluster centre.

It is worth noting that a necessary condition to form such relics is that the initial state of the fossil radio plasma is characterised by a ratio of the magnetic to thermal pressure, $P_{\mathrm{B}} / P_{\text {gas }}$, which has to be as low as 1 per cent to allow shocks to revive $\approx 1 \mathrm{Gyr}$ old radio ghosts. It is also important to mention that Hoeft et al. (2004) find a high probability of radio emission, outside the shocks, due to the drained gas flows induced by the merger events, 

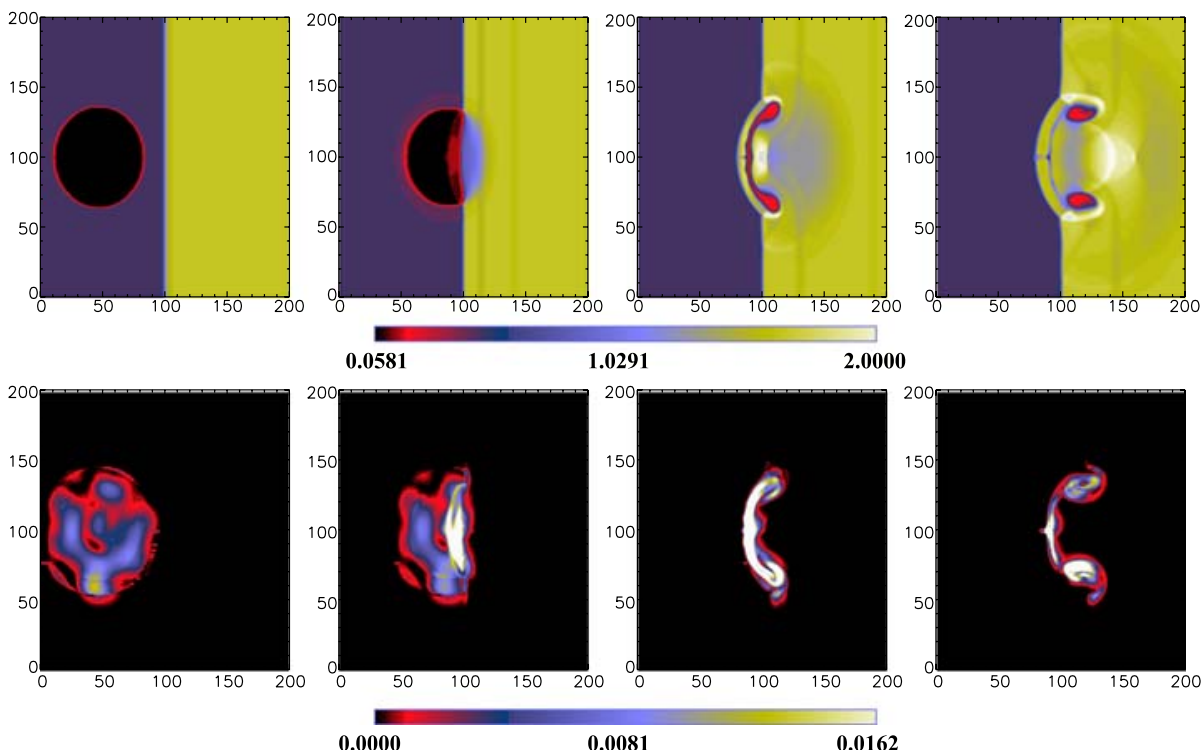

0.0000

0.0081

0.0162

Fig. 11 Results from a simulation of a shock interacting with a fossil radio plasma. The upper (lower) panels show the time evolution of the gas density (magnetic field energy density) during the interaction. From Enßlin and Brüggen (2002)

Fig. 12 Results from a simulation of a galaxy cluster undergoing a major merger. The figure shows the projected 'potential' radio luminosities for 1.13 Gyr old radio plasma, where $P_{B} / P_{\text {gas }}=0.01$. For comparison, the bolometric surface X-ray luminosity (contours) is given. The total bolometric X-ray luminosity of the cluster is $2 \times 10^{44} \mathrm{erg} \mathrm{s}^{-1}$ and the emission-weighted temperature is $3 \mathrm{keV}$. Adapted from Hoeft et al. (2004)

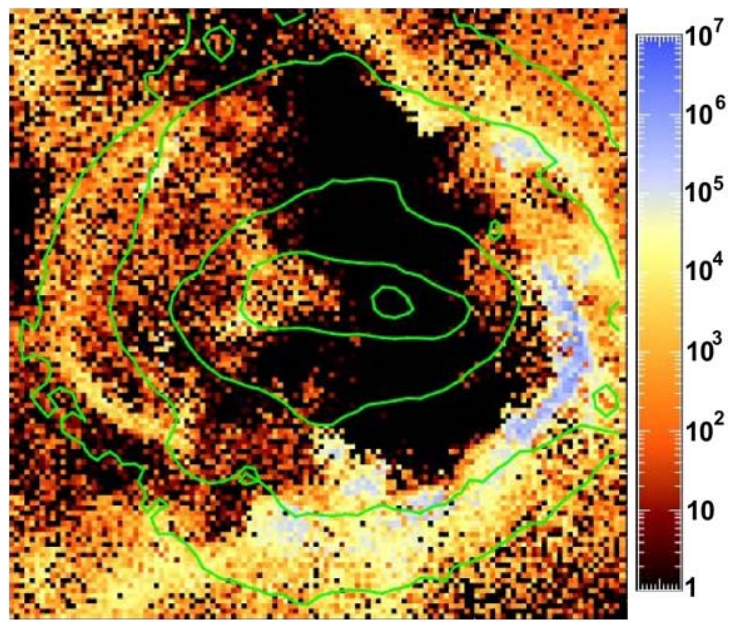

which transport material from the outskirts towards the higher density regions. Therefore in some cases the adiabatic compression seems to be sufficient to revive the fossil radio plasma.

Recently, the picture of diffuse radio emission of galaxy clusters has changed, as more and more detailed observations indicate a more complex picture than the two main phenomena of radio halos and relics (see Ferrari et al. 2008 - Chap. 6, this issue). It can well be that both scenarios described above are at work in galaxy clusters. The in situ re-acceleration of the relativistic particles at shocks might be responsible for the large, external relics, whereas 

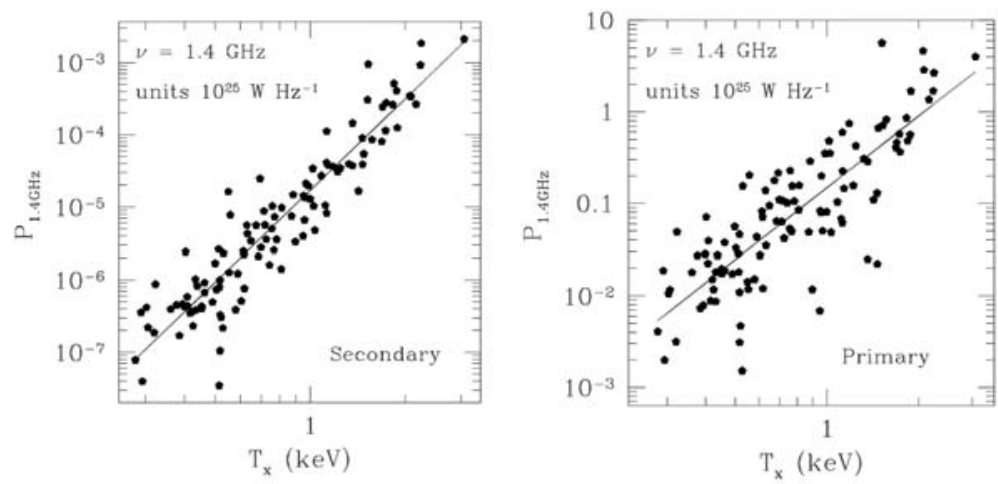

Fig. 13 Predicted radio properties of halos within a cosmological simulation of a cubic volume with a side length of $50 \mathrm{Mpc} / h$, resolved with $256^{3}$ grid cells. The left panel shows the synchrotron power of these halos at $1.4 \mathrm{GHz}$ from secondary electrons. The right panel shows the synchrotron power from primary electrons, mostly located in the peripheral regions. Note that the values for the luminosities due to the primary electrons should be scaled with the electron to proton injection ratio $R_{\mathrm{e}}$ p . From Miniati et al. (2001)

the compression of the fossil radio plasma might lead to the smaller relics which are more disturbed in shape and are usually found closer to the cluster centre.

\subsection{The Quest for Radio Haloes}

The accretion shocks from the LSS formation are also the location of the acceleration of the CRs that will be accreted into the LSS, specially within galaxy clusters. Using the COSMOCR code (Miniati 2001), Miniati et al. (2001) followed primary ions and electrons (injected and accelerated by diffuse cosmic shocks) and secondary electrons and positrons (produced in p-p inelastic collisions of CR ions with thermal ICM nuclei) within a cosmological simulation. Under the assumption that the magnetic field produced by the battery effect reflects a fair representation of the true distribution of the relative magnetic field strengths within the LSS, they were able to predict the central radio emission (radio halos), mainly produced by secondary electrons in a self-consistent treatment (Fig. 13).

Within their modelling they also reproduced the peripheral radio emission (radio relics), mainly produced by primary CRs, where the resulting morphology, polarisation and spectral index match the observed counterparts. However, one has to note that the extrapolation of these simulations (which are on group scale) to the observed data (which are on the scale of massive clusters) might not be straightforward (Fig. 14). It is further worth commenting that even with an electron to proton injection ratio $R_{\mathrm{e} / \mathrm{p}}$ of $10^{-2}$ (which is derived from observations, see Table 5 in Miniati et al. 2001), the predicted luminosities for the primary emission are still significantly higher than for the secondary one (Fig. 14); this prediction does not seem to be confirmed by the observations. Although these results based on a secondary model generally agree with previous work based on simulations of massive galaxy clusters (Dolag and Enßlin 2000), they highlight the general problem that one faces when it is applied the secondary model for relativistic electrons. This model predicts radio haloes for every galaxy cluster, which is in strong contradiction with current observations, as summarised in Fig. 14, which also includes the upper limits obtained by a recent observation campaign at the GRMT (see Brunetti et al. 2007). 


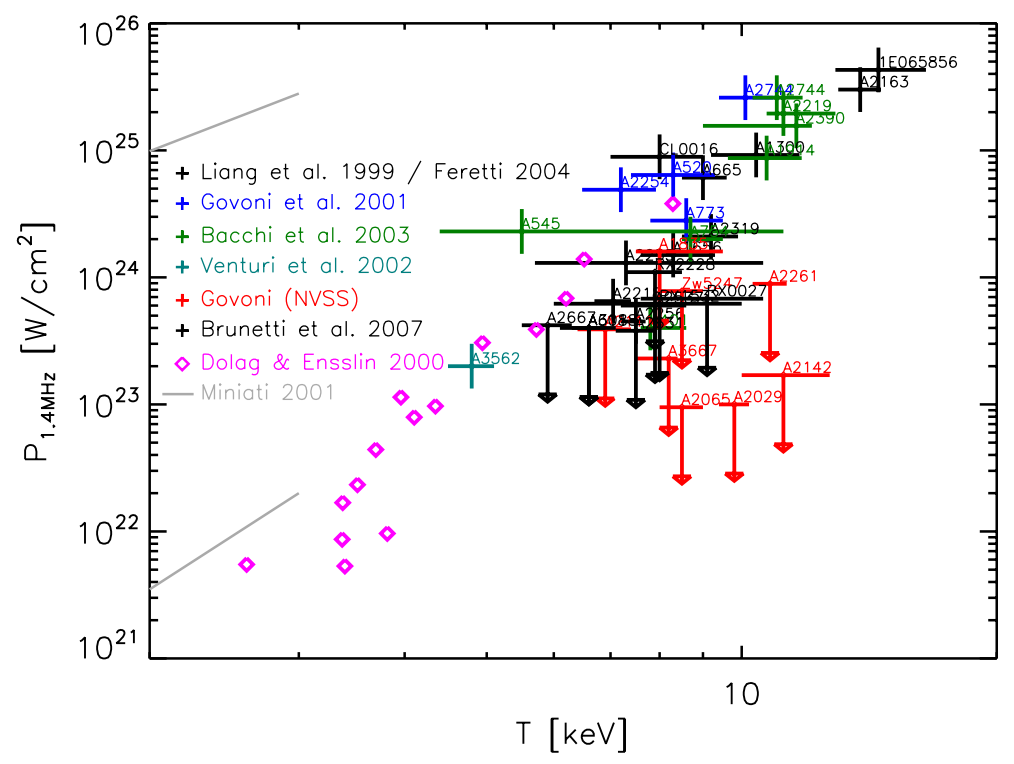

Fig. 14 Total power of radio halos observed at $1.4 \mathrm{MHz}$ vs. cluster temperature. We plot the data from Liang et al. (2000), which were partially re-observed by Feretti (2007, in preparation) together with data from Govoni et al. (2001); Bacchi et al. (2003); Venturi et al. (2003). Some additional upper limits are collected thanks to the help of F. Govoni. Additional upper limits from Brunetti et al. (2007) are shown. We applied a secondary hadronic model, as described in Dolag and Enßlin (2000), to calculate the radio emission from the simulated galaxy clusters. We also added the predictions for the emission from primary (upper line) and secondary (lower line) electrons taken from Miniati et al. (2001) within the temperature range covered by the simulation. Note that the values for the luminosities for primary electrons should be scaled with the electron to proton injection ratio $R_{\mathrm{e} / \mathrm{p}}$

\section{Turbulence}

\subsection{Simulations}

During the growth of the LSS, galaxy clusters continuously accrete other structures, most of the time smaller structures (like galaxies), but sometime also objects with similar mass (major mergers). All these structures continue to move with supersonic or transsonic velocities inside the cluster. Together with the diffuse accretion (which is generally anisotropic) and the generation of turbulence by hydrodynamic instabilities induced by these bulk motions, the gas in clusters of galaxies generally contains an amount of kinetic energy which is not negligible compared to the amount of thermal energy.

It is worth mentioning that different simulation methods reach good agreement in predicting that the ratio of bulk kinetic energy to thermal energy is up to $15 \%$ in galaxy clusters (see Frenk et al. 1999). Based on cosmological hydrodynamic simulations, Inogamov and Sunyaev (2003) pointed out that the broadening of the emission lines in the X-ray band (e.g. the iron $\mathrm{K}$ line) due to these expected bulk motions is appreciably larger than the broadening due to thermal motions. Thus, instruments like the ones designed for Suzaku or future instruments like XEUS will be able to infer such bulk motions from the analysis of the line shapes.

Even after subtraction of the cluster peculiar velocity, large-scale bulk motions will be the main contributor to the deformation of the line shapes. The imprint of turbulence induced 


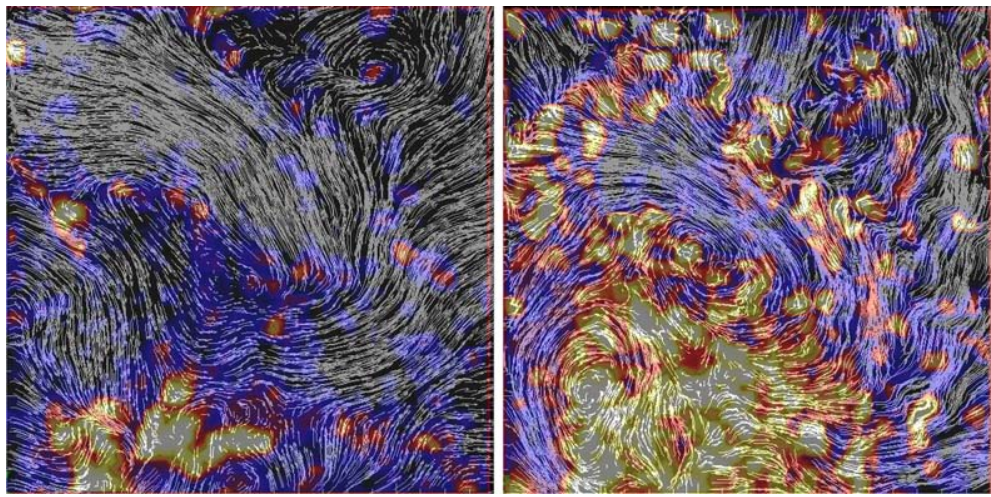

Fig. 15 The gas velocity field in a slice through the central Mpc region of a simulated cluster after subtracting the global mean bulk velocity of the cluster. The left panel shows a run with the original SPH viscosity, the right panel for a low-viscosity scheme. The underlying colour maps represent the ratio of turbulent kinetic energy to total kinetic energy content of the particles, inferred after subtracting the local mean velocity, as described in Dolag et al. (2005b)

by hydrodynamic instabilities along flows (e.g. shear flows) might instead be more subtle to infer from the line profiles.

In recent high-resolution SPH simulations of galaxy clusters within a cosmological environment, Dolag et al. (2005b) used a novel scheme to treat artificial viscosity within the GADGET2 code (Springel 2005) to quantify how intense the shear flow must be to drive fluid instabilities. Being a quite common process within cosmic structure formation, these fluid instabilities driven by shear flows strongly increase the level of turbulence within the ICM (Fig. 15).

Although this small-scale turbulence contributes a measurable effect to the non-thermal pressure within the ICM, the effect on the line shapes is much smaller than the broadening caused by the large-scale bulk motions. Applying this method to a set of simulated galaxy clusters, Vazza et al. (2006) showed that for relaxed clusters the turbulent energy content in the ICM scales with the thermal one. The fraction of turbulent to thermal energy turned out to be rather independent of the mass of the system. Interestingly, this is consistent with the semi-analytical predictions of Cassano and Brunetti (2005), who estimated the amount of turbulent energy within the ICM in terms of the $P \mathrm{~d} V$ work done by the infalling substructures.

\subsection{Observational Evidence}

The spectroscopic resolution of current X-ray instruments is not high enough to measure the line shapes and infer the level of turbulence present within the ICM. However, using a mosaic of XMM-Newton observations of the Coma cluster, Schuecker et al. (2004) were able to produce spatially-resolved gas pressure maps which indicate the presence of a significant amount of turbulence. Performing a Fourier analysis of the data reveals the presence of a scale-invariant pressure fluctuation spectrum in the range between 40 and $90 \mathrm{kpc}$ which is well described by a projected Kolmogorov turbulence spectrum (see Fig. 16); at least 10 percent of the total ICM pressure is in turbulent form.

Alternatively, in a more indirect way, Enßlin and Vogt (2006) argue that the recently reported Kolmogorov-like magnetic turbulence spectrum, which is inferred from Faraday 
Fig. 16 The dots with error bars are the observed projected power spectral densities as inferred from X-ray observations of the Coma cluster, after subtraction of the shot noise; the dashed lines are model predictions. From Schuecker et al. (2004)

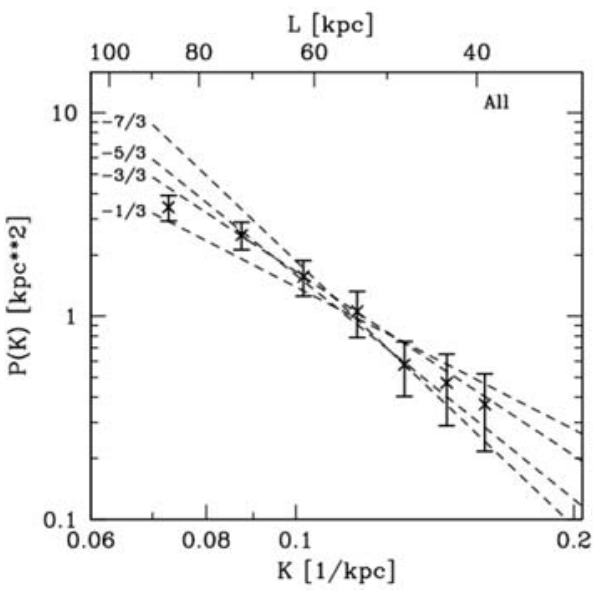

rotation measurements in the cool core of clusters, can be understood by kinetic energy injection (see also Subramanian et al. 2006). Such a dynamo model predicts the correct magnetic field strength in cool core clusters for reasonable (but yet not directly observable) values of the hydrodynamic turbulence velocity and characteristic length scales. This result indicates that the magnetic fields might directly reflect the presence of hydrodynamic turbulence. Such models are directly related to the turbulence induced by buoyant radio lobes from the central radio galaxy which rise into the cluster atmosphere. On the other hand, Faraday rotation within non-cooling flow clusters with multiple extended radio sources (which therefore probe the magnetic field structure at different radii) can give alternative constraints on the magnetic field power spectra (see Murgia et al. 2004; Govoni et al. 2006) and thus on the underlying hydrodynamic turbulence present in the ICM.

\section{Concluding Remarks}

It seems that, in the last years, a consistent picture of magnetic fields in clusters of galaxies has emerged from both numerical work and observations. Simulations of individual processes like shear flows, shock/bubble interactions or turbulence/merging events predict a super-adiabatic amplification of magnetic fields. It is worth mentioning that this common result is obtained by using a variety of different codes, which are based on different numerical schemes. Further support for such super-adiabatic amplifications comes from analytical estimates of the anisotropic collapse which use the Zel'dovich approximation (Zel'dovich 1970). When this amplification occurs in fully consistent cosmological simulations, various observational aspects are reproduced (e.g. Fig. 8); moreover, the final strength of the magnetic field reaches a level sufficient to link models that predict magnetic field seed at high redshift with the magnetic fields observed in galaxy clusters today. It is important to note that all the simulations show that this effect increases when the resolution is improved, and therefore all the current values have to be taken as lower limits of the possible amplification.

Despite this general agreement, there are significant differences among the predictions on the structure of the magnetic fields coming from different models of magnetic field seeds. In particular, there are several differences between the up-scaled cosmological battery fields (Miniati et al. 2001; Sigl et al. 2004) and the magnetic field predicted by the high-resolution 
simulations of galaxy clusters that use either AMR (Brüggen et al. 2005) or SPH (Dolag et al. 1999, 2002, 2005a). In the SPH case, it is possible to follow the amplification of the field seeds within the turbulent ICM in more detail.

A good visual impression can be obtained by comparing the regions filled with the high magnetic fields shown in Figs. 6 and 9. It is clear that the high magnetic field regions for the battery fields are predicted to be much more extended; this result leads to a flat profile around the forming structure, whereas for the turbulent amplified magnetic fields the clusters show a magnetic field distribution which is much more peaked. Part of this difference originates from the physical model, as the cosmological shocks are much stronger outside the clusters. Somewhat less clear is how big it is the influence of the different numerical resolutions of these simulations to these discrepancies. Usually, the model parameters for such simulations can only be calibrated using the magnetic field strength and structure within the high-density regions of galaxy clusters. Therefore it is crucial to perform detailed comparisons with all the available observations to validate the simulations. Note that extrapolating the predictions of the simulations into lower density regions, where no strong observational constraints exist, will further amplify the differences among the simulations in the predictions of the magnetic field structure.

Moreover, one has to keep in mind that, depending on the ICM resistivity, the magnetic field could suffer a decay, which is so far neglected in all the simulations. Furthermore, a clear deficiency of the current simulations is that they do not include the creation of a magnetic field due to all the feedback processes happening within the LSS (like radio bubbles inflated by AGN, galactic winds, etc.): this might alter the magnetic field prediction if their contribution turns out to be significant. Also all of the simulations so far neglect radiative losses; if included, this would lead to a significant increase of the density in the central region of clusters and thereby to a further magnetic field amplification in these regions. Finally, there is an increasing number of arguments suggesting that instabilities and turbulence on very small scales can amplify the magnetic fields to the observed $\mu \mathrm{G}$ level over a relatively short timescale. However, it is still unclear how such fields, which are tangled on very small scales, can be further processed to be aligned on large scales (up to hundreds of kpc), as observed.

Concerning radio haloes and relics, the picture is only partially consistent (for a more detailed discussion of primary and secondary models see Brunetti (2004) and references therein as well as Ferrari et al. 2008-Chap. 6, this issue). Radio relics seem to be most likely related to strong shocks produced by major merger events and therefore produced by direct re-acceleration of CRs, the so-called primary models. Although some of the observed features, like morphology, polarisation and position with respect to the cluster centre, can be reasonably well reproduced, there might be still some puzzles to be solved. On the one hand, direct acceleration of CRs in shocks seems to overestimate the abundance, and maybe the luminosity, of radio relics; on the other hand, simulations which illuminate fossil radio plasma can produce reasonable relics only starting from a small range of parameter settings.

A similar situation arises for modelling the central radio emission of galaxy clusters by secondary models. The total luminosity seems to be reproduced using reasonable assumptions and also the observed steep correlation between cluster temperature/mass and radio power seems to be reproduced quite well. However, these models suffer from two drawbacks. The first one is that, in the framework of such models, every massive cluster produces a powerful radio halo, at odds with observations (Fig. 14). The other problem of secondary models is that the detailed radio properties are not reproduced. In fact, firstly, in most cases, the profile of radio emission is too steep, so that these models can almost never reproduce the size of the observed radio halos. Secondly, the observed spectral steepening (e.g. Giacintucci et al. 2005) cannot be reproduced. 
One possible way out, at least for the first concern, would be that magnetic fields are extremely dynamical and transient and therefore light up the halo during a merger. However, there is also no indication from observations that clusters which show radio emission contain magnetic fields which are more intense than those in clusters without observable extended diffuse radio emission. On the contrary, the cluster A 2142 has a magnetic field strength similar to the Coma cluster, but the upper limit on its radio emission is at least two orders of magnitude below the value expected from the correlation (Fig. 14). Note that both clusters are merging systems which are characterised by the presence of two central cD galaxies. This indicates that there should be further processes involved or additional conditions required to produce radio emission. It is worth noting that recent models, based on turbulent acceleration, seem to overcome this problem (see Kuo et al. 2003; Brunetti and Blasi 2005; Cassano and Brunetti 2005, and references therein). Cassano and Brunetti (2005) predicted that the probability for a galaxy cluster to show a giant radio halo is an increasing function of the cluster mass; these authors also reproduced the observed probability of $\approx 30 \%$ for a massive galaxy clusters to show extended radio emission.

Being dominated by large-scale bulk motions, induced by the cosmological structure formation process, different simulation methods reach good agreement in predicting that the ratio of the bulk kinetic energy to thermal energy has an upper limit of $15 \%$ in galaxy clusters (see Frenk et al. 1999). However, the exact role of turbulence induced by hydrodynamic instabilities within the complex flow patterns of galaxy clusters is still hard to quantify (see Dolag et al. 2005b). It should also be mentioned that the real viscosity of the ICM is hardly known, and can play a noticeable role within the ICM, as demonstrated in simulations which attempt to include physically motivated viscosity into a cosmological context (see Sijacki and Springel 2006).

The dynamical role of CRs in galaxy clusters is not very well understood yet. Simulations using different codes predict quite different relative pressure contained in CRs within galaxy clusters. Moreover, the simulations indicate that the relative importance of CRs in galaxy clusters also strongly depends on other non-thermal processes, like radiative losses and feedback from star formation (see Pfrommer et al. 2007).

In general it has to be pointed out that, although the complexity of the physical processes treated by cosmological simulations have improved dramatically in recent years, this research field is still young. Therefore future work is expected to enlighten such complex processes within the ICM and will help to improve our understanding of the non-thermal components in galaxy clusters. Such work will also be needed to interpret the observational information which is overwhelmingly increasing in quality and size and is expected to become available soon with the next generation of instruments, particularly at radio band, like ALMA, LOFAR, SKA, EVLA and others.

Acknowledgements The authors thank ISSI (Bern) for support of the team "Non-virialized X-ray components in clusters of galaxies". Special thanks to the anonymous referee for suggestions that improved the manuscript, Torsten Enßlin for various helpful discussions and Daniel Clarke for carefully reading the manuscript. A.M.B. acknowledges the support from RBRF grant 06-02-16844 and a support from RAS Programs. A.D. also gratefully acknowledges partial support from the PRIN2006 grant "Costituenti fondamentali dell'Universo" of the Italian Ministry of University and Scientific Research and from the INFN grant PD51.

\section{References}

M. Bacchi, L. Feretti, G. Giovannini, F. Govoni, Astron. Astrophys. 400, 465 (2003)

A.R. Bell, S.G. Lucek, Mon. Not. R. Astron. Soc. 321, 433 (2001)

V.S. Berezinsky, P. Blasi, V.S. Ptuskin, Astrophys. J. 487, 529 (1997) 
S. Bertone, C. Vogt, T. Enßlin, Mon. Not. R. Astron. Soc. 370, 319 (2006)

J. Binney, G. Tabor, Mon. Not. R. Astron. Soc. 276, 663 (1995)

G.T. Birk, H. Wiechen, A. Otto, Astrophys. J. 518, 177 (1999)

D. Biskamp, Magnetohydrodynamic Turbulence (Cambridge University Press, Cambridge, 2003)

R. Blandford, D. Eichler, Phys. Rep. 154, 1 (1987)

M. Brüggen, M. Ruszkowski, A. Simionescu, M. Hoeft, C. Dalla Vecchia, Astrophys. J. 631, L21 (2005)

G. Brunetti, J. Korean Astron. Soc. 37, 493 (2004)

G. Brunetti, P. Blasi, Mon. Not. R. Astron. Soc. 363, 1173 (2005)

G. Brunetti, T. Venturi, D. Dallacasa et al., Astrophys. J. 670, L5 (2007)

M. Bruni, R. Maartens, C.G. Tsagas, Mon. Not. R. Astron. Soc. 338, 785 (2003)

A.M. Bykov, Space Sci. Rev. 99, 317 (2001)

A. Bykov, K. Dolag, F. Durret, Space Sci. Rev. (2008). doi:10.1007/s11214-008-9312-9

R. Cassano, G. Brunetti, Mon. Not. R. Astron. Soc. 357, 1313 (2005)

E. Churazov, W. Forman, C. Jones, H. Böhringer, Astrophys. J. 590, 225 (2003)

T.E. Clarke, P.P. Kronberg, H. Böhringer, Astrophys. J. 547, L111 (2001)

K. Dolag, M. Bartelmann, H. Lesch, Astron. Astrophys. 348, 351 (1999)

K. Dolag, M. Bartelmann, H. Lesch, Astron. Astrophys. 387, 383 (2002)

K. Dolag, T.A. Enßlin, Astron. Astrophys. 362, 151 (2000)

K. Dolag, S. Schindler, F. Govoni, L. Feretti, Astron. Astrophys. 378, 777 (2001)

K. Dolag, D. Grasso, V. Springel, I. Tkachev, J. Exp. Theor. Phys. Lett. 79, 583 (2004)

K. Dolag, D. Grasso, V. Springel, I. Tkachev, J. Cosm. Astro-Part. Phys. 1, 9 (2005a)

K. Dolag, F. Vazza, G. Brunetti, G. Tormen, Mon. Not. R. Astron. Soc. 364, 753 (2005b)

T.A. Enßlin, P.L. Biermann, P.P. Kronberg, X.-P. Wu, Astrophys. J. 477, 560 (1997)

T.A. Enßlin, M. Brüggen, Mon. Not. R. Astron. Soc. 331, 1011 (2002)

T.A. Enßlin, Gopal-Krishna, in Particles and Fields in Radio Galaxies Conference. ASP Conf. Ser., vol. 250 (2001), pp. 454

T.A. Enßlin, C. Vogt, Astron. Astrophys. 453, 447 (2006)

T.A. Enßlin, C. Pfrommer, V. Springel, M. Jubelgas, Astron. Astrophys. 473, 41 (2007)

L. Feretti, D. Dallacasa, G. Giovannini, A. Tagliani, Astron. Astrophys. 302, 680 (1995)

L. Feretti, D. Dallacasa, F. Govoni et al., Astron. Astrophys. 344, 472 (1999)

C. Ferrari, F. Govoni, S. Schindler, A. Bykov, Y. Rephaeli, Space Sci. Rev. (2008). doi:10.1007/s11214-008-9311-x

C.S. Frenk, S.D.M. White, P. Bode et al., Astrophys. J. 525, 554 (1999)

S.R. Furlanetto, A. Loeb, Astrophys. J. 556, 619 (2001)

S. Gabici, P. Blasi, Astrophys. J. 583, 695 (2003)

L. Gazzola, E.J. King, F.R. Pearce, P. Coles, Mon. Not. R. Astron. Soc. 375, 657 (2007)

S. Giacintucci, T. Venturi, G. Brunetti et al., Astron. Astrophys. 440, 867 (2005)

M. Gitti, B.R. McNamara, P.E.J. Nulsen, M.W. Wise, Astrophys. J. 660, 1118 (2007)

F. Govoni, T.A. Enßlin, L. Feretti, G. Giovannini, Astron. Astrophys. 369, 441 (2001)

F. Govoni, M. Murgia, L. Feretti et al., Astron. Astrophys. 460, 425 (2006)

D. Grasso, H.R. Rubinstein, Phys. Rep. 348, 163 (2001)

G. Gregori, F. Miniati, D. Ryu, T.W. Jones, Astrophys. J. 543, 775 (2000)

M. Hoeft, M. Brüggen, G. Yepes, Mon. Not. R. Astron. Soc. 347, 389 (2004)

N.A. Inogamov, R.A. Sunyaev, Astron. Lett. 29, 791 (2003)

T.W. Jones, D.S. De Young, Astrophys. J. 624, 586 (2005)

H. Kang, D. Ryu, R. Cen, D. Song, Astrophys. J. 620, 21 (2005)

H. Kang, D. Ryu, R. Cen, J.P. Ostriker, Astrophys. J. 669, 729 (2007)

K.-T. Kim, P.P. Kronberg, P.C. Tribble, Astrophys. J. 379, 80 (1991)

E.J. King, P. Coles, Mon. Not. R. Astron. Soc. 365, 1288 (2006)

P.P. Kronberg, H. Lesch, U. Hopp, Astrophys. J. 511, 56 (1999)

R.M. Kulsrud, R. Cen, J.P. Ostriker, D. Ryu, Astrophys. J. 480, 481 (1997)

P.-H. Kuo, C.-Y. Hwang, W.-H. Ip, Astrophys. J. 594, 732 (2003)

H. Liang, R.W. Hunstead, M. Birkinshaw, P. Andreani, Astrophys. J. 544, 686 (2000)

M.A. Malkov, L.O.C. Drury, Rep. Prog. Phys. 64, 429 (2001)

F. Miniati, Comput. Phys. Commun. 141, 17 (2001)

F. Miniati, D. Ryu, H. Kang et al., Astrophys. J. 542, 608 (2000)

F. Miniati, T.W. Jones, H. Kang, D. Ryu, Astrophys. J. 562, 233 (2001)

M. Murgia, F. Govoni, L. Feretti et al., Astron. Astrophys. 424, 429 (2004)

S.T. Myers, S.R. Spangler, Astrophys. J. 291, 52 (1985)

C.A. Norman, D.B. Melrose, A. Achterberg, Astrophys. J. 454, 60 (1995)

V. Pavlidou, B.D. Fields, Astrophys. J. 642, 734 (2006) 
C. Pfrommer, V. Springel, T.A. Enßlin, M. Jubelgas, Mon. Not. R. Astron. Soc. 367, 113 (2006)

C. Pfrommer, T.A. Enßlin, V. Springel, M. Jubelgas, K. Dolag, Mon. Not. R. Astron. Soc. 378, 385 (2007)

V. Quilis, J.M.A. Ibanez, D. Saez, Astrophys. J. 502, 518 (1998)

M.J. Rees, M.C. Begelman, R.D. Blandford, E.S. Phinney, Nature 295, 17 (1982)

Y. Rephaeli, J. Nevalainen, T. Ohashi, A. Bykov, Space Sci. Rev. (2008). doi:10.1007/s11214-008-9314-7

C.S. Reynolds, B. McKernan, A.C. Fabian, J.M. Stone, J.C. Vernaleo, Mon. Not. R. Astron. Soc. 357, 242 (2005)

K. Roettiger, J.O. Burns, J.M. Stone, Astrophys. J. 518, 603 (1999a)

K. Roettiger, J.M. Stone, J.O. Burns, Astrophys. J. 518, 594 (1999b)

C. Rordorf, D. Grasso, K. Dolag, Astropart. Phys. 22, 167 (2004)

D. Ryu, H. Kang, P.L. Biermann, Astron. Astrophys. 335, 19 (1998)

D. Ryu, H. Kang, E. Hallman, T.W. Jones, Astrophys. J. 593, 599 (2003)

S. Schindler, A. Diaferio, Space Sci. Rev. (2008). doi:10.1007/s11214-008-9321-8

P. Schuecker, A. Finoguenov, F. Miniati, H. Böhringer, U.G. Briel, Astron. Astrophys. 426, 387 (2004)

G. Sigl, F. Miniati, T.A. Enssßlin, Phys. Rev. D 70, 043007 (2004)

D. Sijacki, V. Springel, Mon. Not. R. Astron. Soc. 371, 1025 (2006)

V. Springel, Mon. Not. R. Astron. Soc. 364, 1105 (2005)

V. Springel, N. Yoshida, S. White, New Astron. 6, 79 (2001)

J.M. Stone, M.L. Norman, Astrophys. J. Suppl. Ser. 80, 753 (1992a)

J.M. Stone, M.L. Norman, Astrophys. J. Suppl. Ser. 80, 791 (1992b)

K. Subramanian, A. Shukurov, N.E.L. Haugen, Mon. Not. R. Astron. Soc. 366, 1437 (2006)

F. Vazza, G. Tormen, R. Cassano, G. Brunetti, K. Dolag, Mon. Not. R. Astron. Soc. 369, L14 (2006)

T. Venturi, S. Bardelli, D. Dallacasa et al., Astron. Astrophys. 402, 913 (2003)

A. Vladimirov, D.C. Ellison, A. Bykov, Astrophys. J. 652, 1246 (2006)

H.J. Völk, A.M. Atoyan, Astrophys. J. 541, 88 (2000)

H.J. Völk, F.A. Aharonian, D. Breitschwerdt, Space Sci. Rev. 75, 279 (1996)

N. Werner, F. Durret, T. Ohasi, S. Schindler, R.P.C. Wiersma, Space Sci. Rev. (2008). doi:10.1007/s11214-008-9320-9

Y.B. Zel'dovich, Astron. Astrophys. 5, 84 (1970) 